\title{
A three phase interpenetrating continua approach for wave and porous structure interaction
}

\author{
Liang Yang ${ }^{\mathrm{a}}$, Andrew Buchan ${ }^{\mathrm{b}}$, Dimitrios Pavlidis ${ }^{\mathrm{c}}$, Alan Jones ${ }^{\mathrm{c}}$, Paul Smith ${ }^{\mathrm{c}}$, \\ Mikio Sakai ${ }^{\mathrm{d}}$, Christopher Pain ${ }^{\mathrm{c}}$ \\ ${ }^{a}$ Department of Energy and Power, Cranfield University, UK \\ ${ }^{b}$ School of Engineering and Materials Science, Queen Mary University of London, UK \\ ${ }^{c}$ Department of Earth Science and Engineering, Imperial College London, UK \\ ${ }^{d}$ Resilience Engineering Research Centre, School of Engineering, The University of Tokyo, \\ Japan
}

\begin{abstract}
A three phase interpenetrating continua model for the numerical simulation of water waves and porous structure interaction is proposed in this work. In contrast with one-fluid formulation/multi-component methods, each phase has its own characteristics, density, velocity, etc and each point is occupied by all phases, this model requires additional closure laws to describe the interfacial momentum exchange, e.g., via the Ergun's equation. First, the porous structure is modelled as a phase of continua with a penalty force adding on the momentum equation, so the conservation of mass is guaranteed without source terms. Second, the adaptive unstructured mesh modelling with P1DG-P1 elements is employed here to decrease the total number of degree of freedom maintaining the same order of accuracy. Several benchmark problems are used to validate the model, which includes the darcy flow, classical collapse of water column and water column with a porous structure.
\end{abstract}

Keywords: interpenetrating continua, porous structure, fluid-structure interaction, anisotropic mesh adaptivity

Highlight:

- The interpenetrating continua approach for wave and porous structure

Email address: liang.yang@cranfield.ac.uk (Liang Yang)

Preprint submitted to Engineering Computation 
interaction problem.

- The porous structure is modelled as a phase of fluid so that the conservation of mass is automatically satisfied in the porous region.

- Adaptive anisotropic mesh in space is employed to reduce the computational cost.

\section{Introduction}

The design, maintenance, and protection of the offshore and coastal infrastructures is widely recognised as critically important at national and worldwide levels. Porous structure exert resistant forces on the flow, generate flow en5 ergy dissipation in coastal and offshore engineering. Understanding the detailed physical processes of wave interaction with the porous structure and the accurate prediction of the flow processes within and around the porous structure is required. There are many experimental and numerical works on the water wave impact on rigid structures $[1,2,3,4,5]$ and the deformable structure [6], and articulated multibody [7]. There is few experimental work for the porous structure found on the literature $[8,9,10]$. Most of the works for the porous structure and water wave interaction is obtained via numerical modelling [11, 12, 13].

For mathematical modelling the water waves, the most common used Eulerian model is the so-called 'one-fluid' approach [14]. The computational modelling of the water wave is carried out in a similar way to that of the single-phase flow, apart from the consideration of the interface evolution. The interface is fully resolved by interface tracking or capturing methods. The most popular approaches of capturing interface are established by Volume of Fluid [15, 16, 17] and the Level Set method $[3,6]$

Another approach worth mentioning is the particle-based methods, Smoothed Particle Hydrodynamics (SPH) $[18,19]$ or Moving particle semi-implicit (MPS) [20, 21, 22], that has been recently applied to coastal engineering [18, 23]. This approach solves the flow in a Lagrangian framework, solving the kinematics of 
each particle and its interaction with neighbouring particles. The Lagrangian nature of SPH makes it well suited to simulate free surface flows with rapid changes of the flow field.

There are also simplified wave models, such as non-hydrostatic wave model [24] or the potential flow model [25]. The Navier-Stokes equations are greatly simplified, resulting in an explicit equation for free surface evolution. However,

30 this model is not able to simulate the discontinuous free surface, such as breaking waves

The presence of flow through a porous structure is often modelled as a drag and inertia terms with empirical parameters on the macroscopic scale $[11,12$, 13]. On the other hand, the microscopic approach can fully resolve the flow within the porous structure with the flow resolution being at the pore scale [26]. However this requires large computational resources and is impractical for offshore and coastal engineering.

Works prior to the one-fluid approaches include the interpenetrating continua approach which uses an averaged mixture fluid model. Such methods are used extensively in $\mathrm{n}[27]$, chemical reactors $[28,29]$ and combustion modellings [30]. Different phases or components in a multiple-fluid flow have different fluid properties (e.g. density, viscosity etc.), and as a result, they move at different velocities, causing relative motions between phases or components. We introduce an interpenetrating model for simulating water wave and porous structure, in 45 which the distribution of different phases or components is represented by their volume fractions and does not rely on continuous tracking of interfaces. The porous structure is fixed by a large penalty drag force added on the structure's inertia term. Another advantage of using the interpenetrating continua model are that the movement, fracture, and break of the porous structure, porosity changes can be easily embedded for further complex studies.

The structure of this paper is organised as follows. Section 2 introduces the classical Eulerian conservation laws (conservation of linear momentum and mass) for multiple mixtures of fluids, by considered each fluid separately. Section 3 describes the details of numerical discretisation of the multiphase flow 
governing equations. An efficient P1DG-P1 scheme set in an Eulerian unstructured mesh is chosen for the spatial discretisation in conjunction with a well established fractional step method for the fluid-pressure decoupling. Section 4 presents numerical problems, illustrating the capability of the proposed method.

\section{Governing equations}

60 Derivation of the interpenetrating continua model can be found in [31]. In the following, we will write the equation governing the evolution of the mass factions $\alpha_{i}, i=1, \ldots, n$.

\subsection{Conservation of mass}

If there is no mass transfer between each phases.

$$
\frac{\partial}{\partial t}\left(\alpha_{i} \rho_{i}\right)+\nabla \cdot\left(\alpha_{i} \rho_{i} \boldsymbol{u}_{i}\right)=0, \quad 1 \leqslant i \leqslant n
$$

Assuming the three phases are all incompressible, we have mass conservation equation

$$
\frac{\partial \alpha_{i}}{\partial t}+\nabla \cdot\left(\alpha_{i} \boldsymbol{u}_{i}\right)=0, \quad 1 \leqslant i \leqslant n .
$$

Adding up all phases, we have

$$
\nabla \cdot\left(\sum_{i}^{n} \boldsymbol{u}_{i}\right)=0
$$

which is the global mass conservation equation.

\subsection{Conservation of linear momentum}

For multiphase problem, the conservation of linear momentum equation can be written as

$$
\alpha_{i} \rho_{i} \frac{\partial \boldsymbol{u}_{i}}{\partial t}+\alpha_{i} \rho\left(\boldsymbol{u}_{i} \cdot \nabla\right) \boldsymbol{u}_{i}=-\alpha_{i} \nabla p_{i}+\nabla \cdot \boldsymbol{\sigma}_{i}^{\prime}+\boldsymbol{f}_{i}+\alpha_{i} \rho_{i} \boldsymbol{g}
$$

where $\boldsymbol{\nabla} \cdot \boldsymbol{\sigma}^{\prime}=\mu_{i} \Delta^{2} \boldsymbol{u}_{i}+\frac{1}{3} \mu_{i} \boldsymbol{\nabla}\left(\boldsymbol{\nabla} \cdot \boldsymbol{u}_{i}\right)$ is the viscous force and $p$ is the pressure, $\boldsymbol{f}_{i}$ is the drag force between phase $i$ and $j$ and will be discussed in section 2.4. 


\subsection{Equation of State}

In general, to complete the sets of governing equations, it is necessary to introduce an equation of state relating density to pressure. However, in the proposed wave and porous structure interaction model, the three phases are assumed to be incompressible with a constant density.

\subsection{Inter-phase momentum transfer}

The interfacial momentum transfer is crucial to the modelling of multiphase flows. Considered as sources or sinks in the momentum equations, this interfacial force density generally contains the force due to the viscous drag as well as virtual mass and turbulent dispersion which are lumped together as non-drag forces. These interfacial force densities strongly govern the distribution of the volume fraction. The drag forces between the multiple phases are the most important coupling forces.

$$
\Sigma_{l g}=\frac{3}{4} C_{D} \frac{\alpha_{l g} \alpha_{g l} \rho_{l}\left|\boldsymbol{u}_{g}-\boldsymbol{u}_{l}\right|}{d_{p}} \alpha_{l g}^{-2.65}
$$

The interfacial drag force components can be modelled according to the interfacial drag force vector $\boldsymbol{f}$, where $\boldsymbol{u}_{g}, \boldsymbol{u}_{l}$ and $\boldsymbol{u}_{s}$ are unknown variables

$$
\boldsymbol{f}=\left\{\begin{array}{cl}
\Sigma_{l g}\left(\boldsymbol{u}_{g}-\boldsymbol{u}_{l}\right)+\Sigma_{s l}\left(\boldsymbol{u}_{s}-\boldsymbol{u}_{l}\right) & \text { liquid phase } \\
\Sigma_{l g}\left(\boldsymbol{u}_{l}-\boldsymbol{u}_{g}\right)+\Sigma_{s g}\left(\boldsymbol{u}_{s}-\boldsymbol{u}_{g}\right) & \text { gas phase } \\
\infty & \text { solid phase }
\end{array}\right.
$$

Assume the liquid and solid phase is a continuous phase and gas are particulate phase, the inter-facial drag coefficients are (from Ergun equation [32, 33, 34]), where the first terms is linear with the velocity, corresponding to the viscous effect, the second term in quadratic form of velocity represents the inertia effect:

$$
\begin{aligned}
& \Sigma_{s g}=150 \frac{\alpha_{g s}^{2} \mu_{g}}{\alpha_{g s} d_{p}^{2}}+1.75 \frac{\alpha_{g s} \rho_{g}\left|\boldsymbol{u}_{g}-\boldsymbol{u}_{s}\right|}{d_{p}} \\
& \Sigma_{s l}=150 \frac{\alpha_{l s}^{2} \mu_{g}}{\alpha_{s l} l_{p}^{2}}+1.75 \frac{\alpha_{g s} \rho_{l}\left|\boldsymbol{u}_{l}-\boldsymbol{u}_{s}\right|}{d_{p}} \\
& \Sigma_{l g}=\frac{3}{4} C_{D} \frac{\alpha_{l g} \alpha_{g l} \rho_{l}\left|\boldsymbol{u}_{g}-\boldsymbol{u}_{l}\right|}{d_{p}} \alpha_{l g}^{-2.65}
\end{aligned}
$$


where $d_{p}$ is the effective mean particle diameters.For the interfacial drag between liquid and gas phases, the interface drag coefficients based on the correlations by Ishii and Zuber [35] for different flow regimes are normally employed for gasliquid flows. The drag curve $C_{D}$ can be correlated for individual bubbles across several distinct bubble Reynolds number regions:

$$
C_{D}= \begin{cases}\frac{24}{\alpha_{l g} R e_{l g}}\left[1+0.15\left(\alpha_{l g} R e_{l g}\right)^{0.687}\right], & \text { if } \alpha_{l g} R e_{l g} \leqslant 1000 \text { viscous region } \\ 0.44 & \text { if } \alpha_{l g} R e_{l g}>1000 \text { turbulent region }\end{cases}
$$

$$
R e_{l g}=\frac{\rho_{l}\left|\boldsymbol{u}_{g}-\boldsymbol{u}_{l}\right| d_{b}}{\mu_{l}}
$$

The average bubble diameter is calculated using

$$
d_{b}=\frac{W_{e} \sigma}{\rho_{l}\left(\boldsymbol{u}_{g}-\boldsymbol{u}_{l}\right)^{2}}
$$

The normalised volume fraction is calculated as,

$$
\alpha_{i j}=\frac{\alpha_{i}}{\alpha_{i}+\alpha_{j}}
$$

where $\alpha_{i j}$ is the normalised volume fraction of phase $i$ in phase $j$.

\section{Numerical scheme}

The spatial discretisation is based on the control volume finite element method. The details of the numerical finite element discretisation and solution of these equations are given [16]. Here, triangular meshes (2D) tetrahedral (3D) are used to mesh the domain as illustrated in Fig. 1. In summary, we use the linear discontinuous between elements velocity and linear continuous pressure (P1DG-P1) for spatial discretisation. This model has been incorporated into the general purpose CFD code FLUIDITY. A high order discretisation in time

90 is used based on Crank-Nicholson time stepping. The Courant-Friedirichs-Lewy (CFL) condition is adopted for determining the time step. 


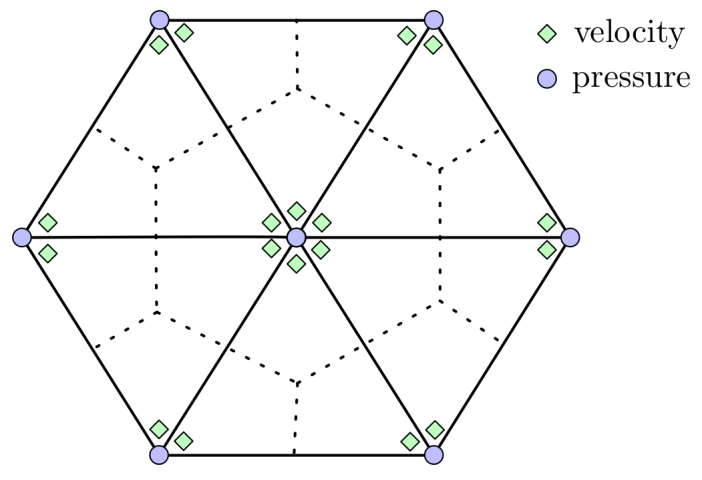

Figure 1: Finite element used to discretise the governing equations. The central position of key solution variables (velocity and pressure) are indicated here for the P1DG-P1 pairs in 2D. 


\section{Numerical examples}

In this section, the numerical algorithm and implementation is benchmarked with the experimental data.

1.

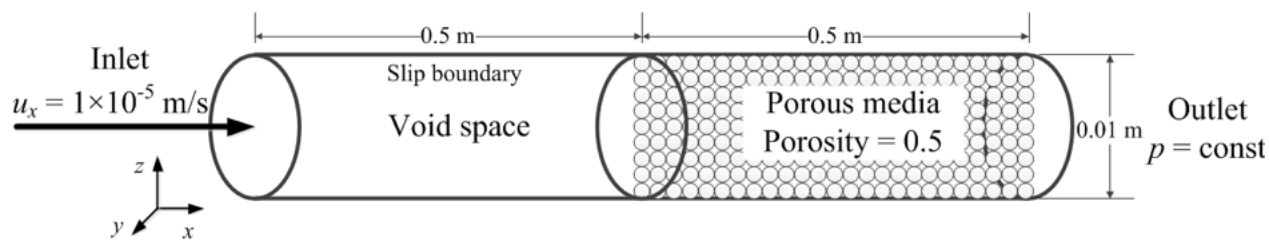

Figure 2: Schematic of fluid flowing from the void space to the porous media with constant velocity.

\begin{tabular}{cccccc}
\hline Density & Viscosity & Porosity & Permeability & Outlet Pressure & Inlet Velocity \\
\hline $1000 \mathrm{~kg} / \mathrm{m}^{3}$ & $1.0 \mathrm{cp}$ & 0.5 & 0.1 & $10^{5} \mathrm{~Pa}$ & $10^{-5} \mathrm{~m} / \mathrm{s}$ \\
\hline
\end{tabular}

Table 1: Physical properties used for the modelling.

Since the slip boundary condition is applied to the void space section, the pressure drop is neglected. The analytical pressure drop in the porous media is calculated using the Darcy equation:

$$
\frac{q}{A}=v=-\frac{\kappa}{\mu} \frac{d p}{d x}
$$

where $q$ is the volumetric flow rate and $A$ is the area of cross section. $v$ is the Darcy velocity, $k$ is the permeability and $\mu$ is the viscosity, $p$ is the pressure and $x$ is the coordinate. 
The comparison results of the axial pressure with different mesh refinements are shown in Fig. 3. It can be seen that the axial pressures of different grid sizes are similar, and the modelling results show good agreements with the analytical solution. Thus, the proposed approach can give relatively accurate results for the modelling of a free flow into the porous media.

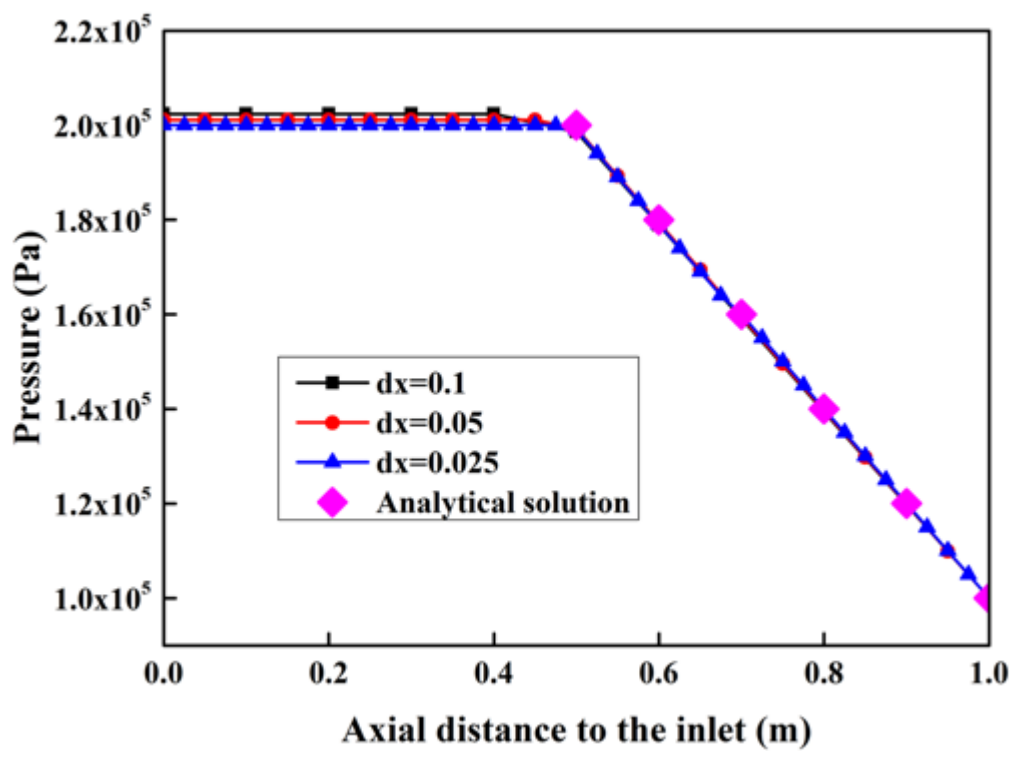

Figure 3: Comparison between the results of mesh refinements and analytical solution.

\subsection{D collapsing water column}

The second numerical example verifies the capability of multi-fluid model for the numerical simulation of free surface flow. The dam break problem is a well documented example at experimental [36] and numerical [6] level, which simulates the sudden collapse of a square shaped column of water onto a horizontal surface as a result of the effect of gravity. The general description of the problem is presented in Figure 4. For the numerical results presented herein, the side of the (square) water domain is initially prescribed as $a=1 \mathrm{~m}$ (see Figure 4). The water phase is fully embedded inside a rectangular domain of base length $b=5 \mathrm{~m}$, height was chosen as $h=1.25 \mathrm{~m}$. Non-slip boundary 


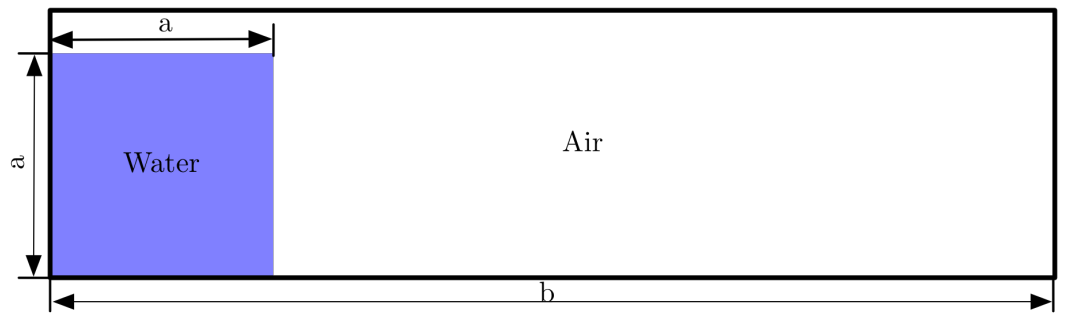

Figure 4: Schematics of the initial conditions for the collapsing water column $(a=1 \mathrm{~m}$; $b=5 \mathrm{~m})$.

conditions are considered for all the sides of the rectangular domain. The fluid properties of both phases (water and air will be referred by the subscripts $w$ and $a$, respectively) are listed in Table 2 below.

\begin{tabular}{llll}
\hline water density & air density & water viscosity & air viscosity \\
\hline$\rho_{w}=1000 \mathrm{~kg} / \mathrm{m}^{3}$ & $\rho_{a}=1 \mathrm{~kg} / \mathrm{m}^{3}$ & $\mu_{w}=10^{-3} \mathrm{~Pa} \mathrm{~s}$ & $\mu_{a}=10^{-5} \mathrm{~Pa} \mathrm{~s}$ \\
\hline
\end{tabular}

Table 2: Physical properties of water and air for collapsing water column problem.

Figure 5 illustrates a sequence of snapshots of the free surface position as a function of time. Note that an interpenetrating continua model is used, so we take the contour line of volume fraction showing the implicit interface. The predicted heights and the surge front location of the collapsed water are plotted against the dimensionless time $\tau=t \sqrt{h / g}$, as reported in [36]. In Figure 6 , very good agreement can be observed between the numerical simulation obtained using the proposed algorithm and the experimental [36] available in the literature. As can be observed, with mesh refinement, the presented results converge extremely well to the latest experimental data.

Overall, the comparison between the numerical results, the interpenetrating fluid numerical formulation and the experimental date indicate that the present numerical model is capable of simulating the hydrodynamics of water and air. 


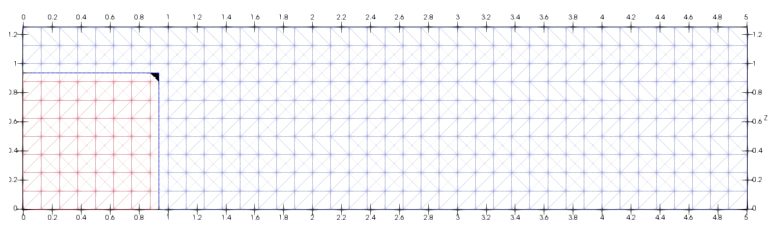

(a) $\tau=0.0$

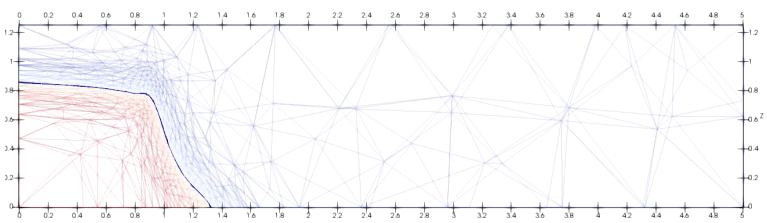

(b) $\tau=0.6756$

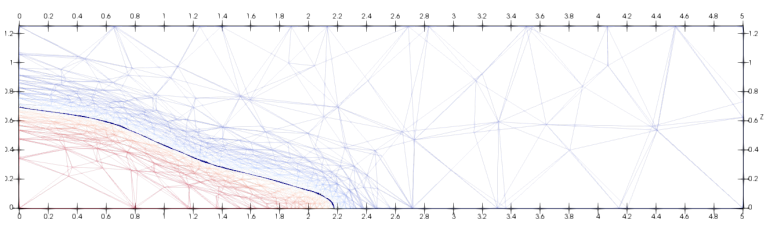

(c) $\tau=1.4503$

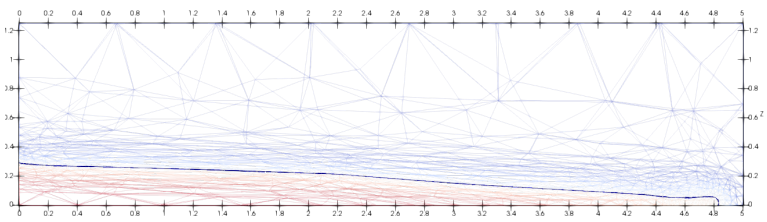

(d) $\tau=3.8403$

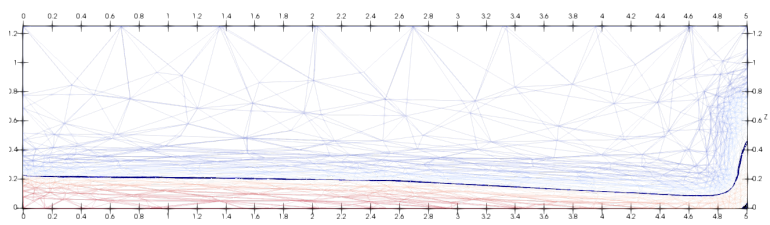

(e) $\tau=4.7086$

Figure 5: Predicted free surface evolution at different dimensionless time steps $\tau$ obtained for a adaptive mesh. (a) $\tau=0.0$; (b) $\tau=0.6756$; (c) $\tau=1.4503$; (d) $\tau=3.8403$; (e) $\tau=4.7086$. The material parameters are as follows: water density $\rho_{w}=1000 \mathrm{~kg} / \mathrm{m}^{3}$, air density $\rho_{a}=1$ $\mathrm{kg} / \mathrm{m}^{3}$, water viscosity $\mu_{w}=10^{-3} \mathrm{~Pa} \mathrm{~s}$, air viscosity $\mu_{a}=10^{-5} \mathrm{~Pa}$ s (refer to Figure 4 and Table 2). 


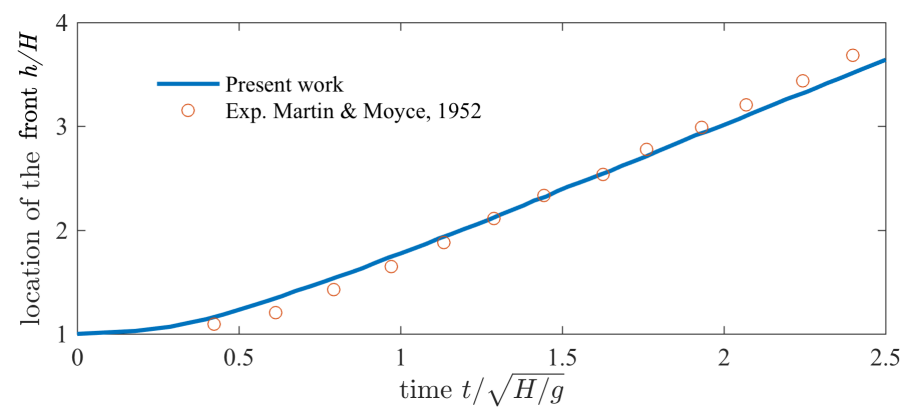

(a): surge front position

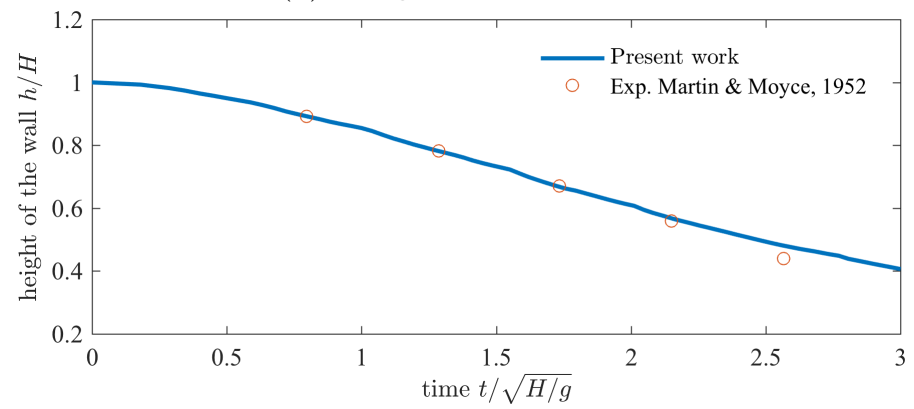

(b): water column height

Figure 6: Comparison of the surge front location and the water column height with experimental data and numerical results. The corresponding dimensionless time refinement is as follows: $\Delta \tau=8 \times 10^{-4}, \Delta \tau=4 \times 10^{-4}$ and $\Delta \tau=2 \times 10^{-4}$. The material parameters are as follows: water density $\rho_{w}=1000 \mathrm{~kg} / \mathrm{m}^{3}$, air density $\rho_{a}=1 \mathrm{~kg} / \mathrm{m}^{3}$, water viscosity $\mu_{w}=10^{-3} \mathrm{~Pa} \mathrm{~s}$, air viscosity $\mu_{a}=10^{-5} \mathrm{~Pa} \mathrm{~s}$ (refer to Figure 4 and Table 2). 
The second numerical example is performed to verify the drag force by comparing the computational results with the experiment. Lin [8] tested a dam break wave through different porous materials. The tank is $0.89 \mathrm{~m}$ long and $0.555 \mathrm{~m}$ height, in the middle there is a porous block of $0.29 \mathrm{~m}$, as shown in Fig. 7. Initially the ground water is $3 \mathrm{~cm}$ depth and the water column ext has a $2 \mathrm{~cm}$ gap to the left porous wall. Two different baffle materials were used, crushed rocks and glass beads. In case 1, the porous block is composed by crashed rock with an averaged porosity of 0.49 . In case 2 , the glass beads has a porosity of 0.39. The conservation of the mass is guaranteed. There is no 'lose mass' compared with the 'one-fluid' formulation. The pore-based Reynolds number for the crushed rock experiments is $R e_{p}=325$, while for the glass beads experiments it is $R e_{p}=9.6$.

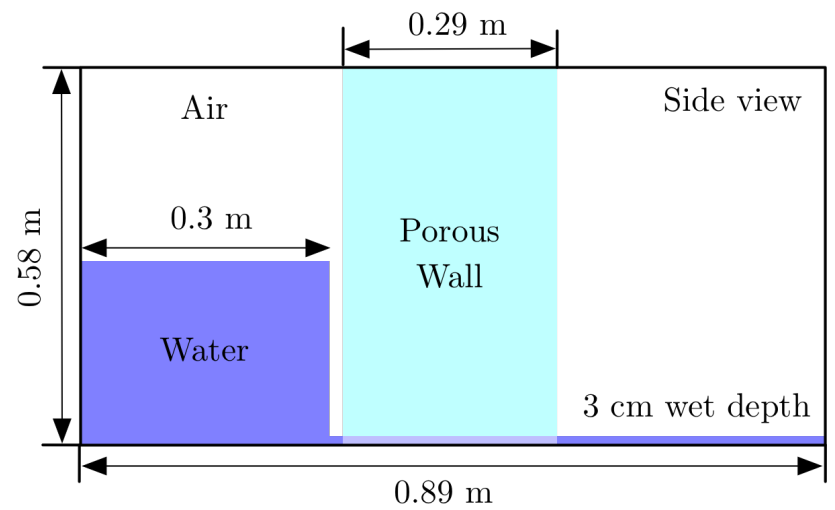

Figure 7: Schematics of the initial condition for the dam break problem.

Fig. 8-9 show representative snapshots for the two cases. The computed and measured free surface time evolution agrees very well for the two porous media tested. 


\begin{tabular}{lll}
\hline & case 1 (crashed rock) & case 2 (glass beads) \\
\hline domain size $(\mathrm{m})$ & $0.89 \times 0.58 \times 0.1$ & \\
water density $\left(\mathrm{kg} / \mathrm{m}^{3}\right)$ & 1000 & \\
air density $\left(\mathrm{kg} / \mathrm{m}^{3}\right)$ & 1 \\
water viscosity $(\mathrm{Pa} \mathrm{s})$ & $1 \times 10^{-3}$ & \\
air viscosity $(\mathrm{Pa} \mathrm{s})$ & $1 \times 10^{-5}$ & \\
baffle position $(\mathrm{m})$ & 0.3 & $0.3 \times 10^{-2}$ \\
baffle thickness $(\mathrm{m})$ & 0.29 & 0.15 \\
average grain size $(\mathrm{m})$ & $1.59 \times 10^{-2}$ & 0.39 \\
initial dam height $(\mathrm{m})$ & 0.25 & 0.49 \\
porosity &
\end{tabular}

Table 3: Computational details of the dam break through a porous structure problem.

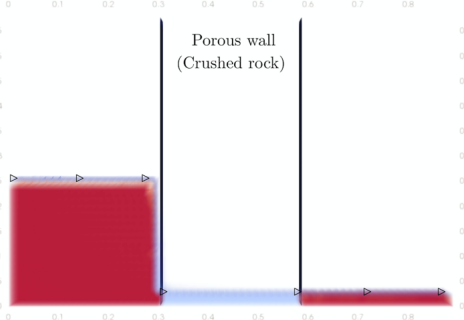

(a)

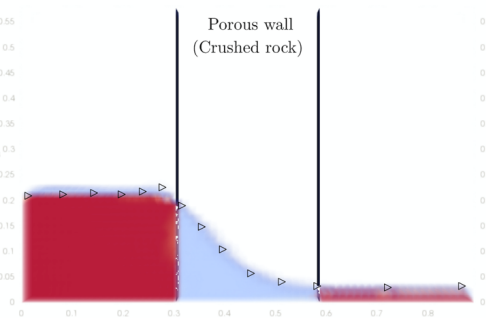

(c)

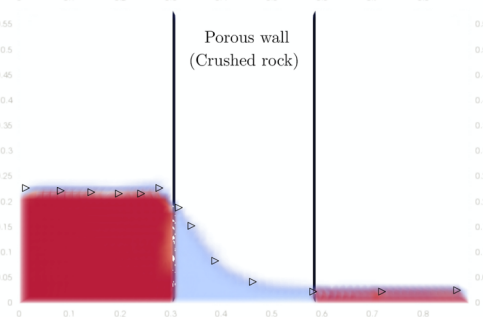

(b)

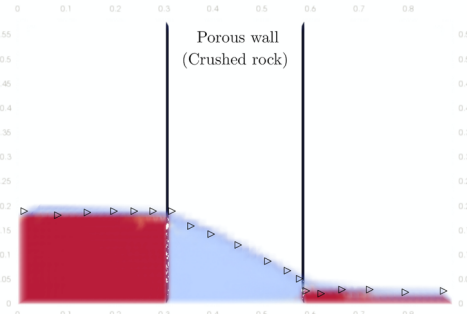

(d)

Figure 8: Comparison of free surface shapes for dam break through crush rock: (a) $t=0.00$ s. (b) $t=0.02 \mathrm{~s}$. (c) $t=0.04 \mathrm{~s}$. (d) $t=0.12 \mathrm{~s}$. Triangle markers is from the experimental data by Liu et al [9]. 


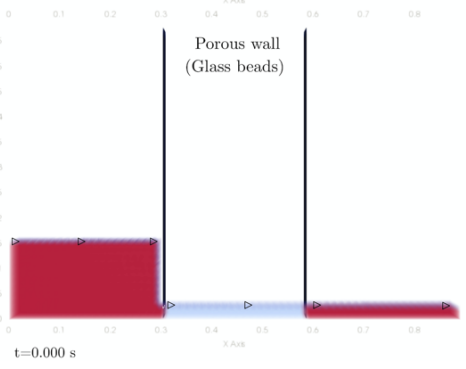

(a)

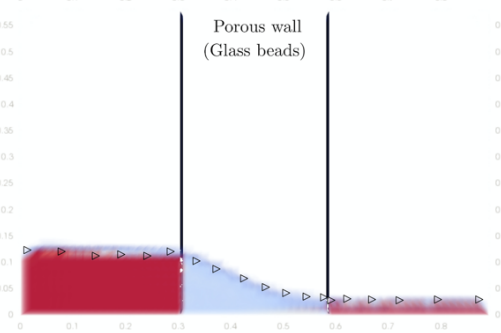

(c)

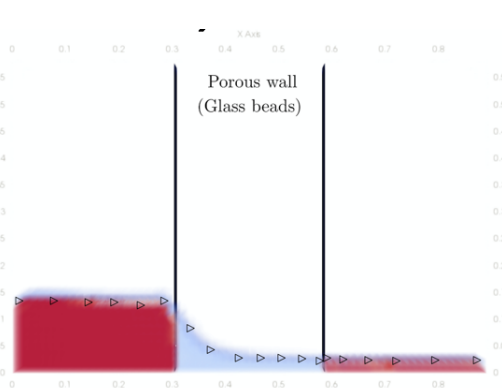

(b)

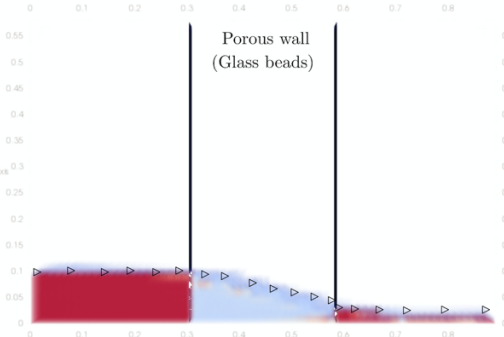

(d)

Figure 9: Comparison of free surface shapes for dam break through glass beads barrier: (a) $t=0.00 \mathrm{~s}$. (b) $t=0.02 \mathrm{~s}$. (c) $t=0.04 \mathrm{~s}$. (d) $t=0.12 \mathrm{~s}$. Triangle markers is from the experimental data by Liu et al [9].

\section{Conclusions}

The contribution of this paper is to bring the interpenetrating continua model for modelling the breaking water wave and porous structure interaction. On the basis of the continua framework, separate transport equations governing the conservation laws are solved for each phase and exchanges that take place at the interfaces are explicitly account for, the dynamics of the interaction between the individual phases can be effectively described via suitable correlation models. These correlation models is well-suited to simulate the macroscopic behaviour of large-scale flows, which do not resolve all the relevant length and timescales. The key success to the application of this model is the reliance on the proper correlation of the inter-phase terms. Several validated cases have been provided 
in the numerical examples to prove the accuracy of the proposed numerical method.

\section{Acknowledgements}

from HEFCE/Research England. A. Buchan acknowledges the support from EPSRC Grant EP/M022684/1.

\section{Reference}

[1] P. Blackmore, P. Hewson, Experiments on full-scale wave impact pressures, Coastal Engineering 8 (4) (1984) 331-346.

[2] F. Dias, J.-M. Ghidaglia, Slamming: Recent progress in the evaluation of impact pressures, Annual Review of Fluid Mechanics 50 (1) (2018) 243-273.

[3] L. Yang, H. Yang, S. Yan, Q. Ma, M. Bihnam, Comparative study on water impact problem, in: The 26th International Ocean and Polar Engineering Conference, International Society of Offshore and Polar Engineers, 2016, pp. 27-34.

[4] L. Yang, One-fluid formulation for fluid-structure interaction with free surface, Computer Methods in Applied Mechanics and Engineering 332 (2018) $102-135$.

[5] L. Chen, D. Stagonas, H. Santo, E. Buldakov, R. Simons, P. Taylor, J. Zang, Numerical modelling of interactions of waves and sheared currents with a surface piercing vertical cylinder, Coastal Engineering 145 (2019) 65-83.

[6] L. Yang, A. J. Gil, A. A. Carreño, J. Bonet, Unified one-fluid formulation for incompressible flexible solids and multiphase flows: Application to hydrodynamics using the Immersed Structural Potential Method (ISPM), International Journal for Numerical Methods in Fluid 86 (1) (2018) 78-106. 
[7] L. Yang, Z. Lyu, P. Yang, D. Pavlidis, F. Fang, J. Xiang, J.-P. Latham, C. Pain, Numerical simulation of Attenuator Wave Energy Converter using one-fluid formulation, in: The 28th International Ocean and Polar Engineering Conference, International Society of Offshore and Polar Engineers, 2018, pp. 602-607.

[8] P. Lin, Numerical modeling of breaking waves, Cornell University, 1998.

[9] P. L. F. Liu, P. Lin, K.-A. Chang, T. Sakakiyama, Numerical modeling of wave interaction with porous structures, Journal of waterway, port, coastal, and ocean engineering 125 (6) (1999) 322-330.

[10] H. Santo, D. Stagonas, E. Buldakov, P. Taylor, Current blockage in sheared flow: Experiments and numerical modelling of regular waves and strongly sheared current through a space-frame structure, Journal of Fluids and Structures 70 (2017) 374-389.

[11] M. del Jesus, J. L. Lara, I. J. Losada, Three-dimensional interaction of waves and porous coastal structures: Part I: Numerical model formulation, Coastal Engineering 64 (2012) 57-72.

[12] J. L. Lara, M. del Jesus, I. J. Losada, Three-dimensional interaction of waves and porous coastal structures: Part II: Experimental validation, Coastal Engineering 64 (2012) 26-46.

[13] K. C. Hu, S. C. Hsiao, H. H. Hwung, T. R. Wu, Three-dimensional numerical modeling of the interaction of dam-break waves and porous media, Advances in Water Resources 47 (2012) 14-30.

[14] L. Yang, An immersed computational framework for multiphase FluidStructure Interaction, Ph.D. thesis, Swansea University (2015).

[15] Z. Xie, D. Pavlidis, J. R. Percival, J. L. Gomes, C. C. Pain, O. K. Matar, Adaptive unstructured mesh modelling of multiphase flows, International Journal of Multiphase Flow 67 (2014) 104-110. 
[16] D. Pavlidis, Z. Xie, J. R. Percival, J. L. Gomes, C. C. Pain, O. K. Matar,

[18] S. Shao, Incompressible SPH flow model for wave interactions with porous media, Coastal Engineering 57 (3) (2010) 304-316.

[19] H. Basser, M. Rudman, E. Daly, SPH modelling of multi-fluid lockTwo-and three-phase horizontal slug flow simulations using an interfacecapturing compositional approach, International Journal of Multiphase Flow 67 (2014) 85-91.

[17] D. Pavlidis, J. L. Gomes, Z. Xie, J. R. Percival, C. C. Pain, O. K. Matar, Compressive advection and multi-component methods for interfacecapturing, International Journal for Numerical Methods in Fluids 80 (4) (2016) 256-282. exchange over and within porous media, Advances in Water Resources 108 (2017) 15-28.

[20] S. Koshizuka, Y. Oka, Moving-particle semi-implicit method for fragmentation of incompressible fluid, Nuclear science and engineering 123 (3) (1996) $421-434$.

[21] K. Takabatake, X. Sun, M. Sakai, D. Pavlidis, J. Xiang, C. C. Pain, Numerical study on a heat transfer model in a Lagrangian fluid dynamics simulation, International Journal of Heat and Mass Transfer 103 (2016) 635-645.

[22] X. Sun, M. Sun, K. Takabatake, C. C. Pain, M. Sakai, Numerical simulation of free surface fluid flows through porous media by using the explicit MPS method, Transport in Porous Media.

[23] Y. Zhou, P. Dong, A new implementation method of sharp interface boundary conditions for particle methods in simulating wave interaction with submerged porous structure, Computers \& Fluids 177 (2018) 87-100.

[24] G. Ma, F. Shi, S.-C. Hsiao, Y.-T. Wu, Non-hydrostatic modeling of wave interactions with porous structures, Coastal Engineering 91 (2014) 84-98. 
[25] S. Yan, Q. Ma, Numerical simulation of fully nonlinear interaction between steep waves and 2D floating bodies using the QALE-FEM method, Journal of Computational Physics 221 (2) (2007) 666-692.

[26] L. Yang, J. Yang, E. Boek, M. Sakai, C. Pain, Image-based simulations of absolute permeability with massively parallel pseudo-compressible stabilised finite element solver, Computational Geosciences 23 (5) (2019) 881893.

[27] C. Pain, C. de Oliveira, A. Goddard, Modelling the criticality consequences of free surface motion in fissile liquids, Tech. rep. (1999).

[28] C. Pain, S. Mansoorzadeh, C. De Oliveira, A. H. Goddard, Numerical modelling of gas-solid fluidized beds using the two-fluid approach, International Journal for numerical methods in fluids 36 (1) (2001) 91-124.

[29] C. Pain, S. Mansoorzadeh, C. De Oliveira, A study of bubbling and slugging fluidised beds using the two-fluid granular temperature model, International Journal of Multiphase Flow 27 (3) (2001) 527-551.

[30] C. Baumgarten, Mixture formation in internal combustion engines, Springer Science \& Business Media, 2006.

[31] R. Temam, A. Miranville, Mathematical modeling in continuum mechanics, Cambridge University Press, 2005.

[32] I. Macdonald, M. El-Sayed, K. Mow, F. Dullien, Flow through porous media-the Ergun equation revisited, Industrial \& Engineering Chemistry Fundamentals 18 (3) (1979) 199-208.

[33] M. Sakai, M. Abe, Y. Shigeto, S. Mizutani, H. Takahashi, A. Viré, J. R. Percival, J. Xiang, C. C. Pain, Verification and validation of a coarse grain model of the DEM in a bubbling fluidized bed, Chemical Engineering Journal 244 (2014) 33-43. 
[34] K. Takabatake, Y. Mori, J. G. Khinast, M. Sakai, Numerical investigation of a coarse-grain discrete element method in solid mixing in a spouted bed, Chemical Engineering Journal 346 (2018) 416-426.

[35] M. Ishii, N. Zuber, Drag coefficient and relative velocity in bubbly, droplet or particulate flows, AIChE Journal 25 (5) (1979) 843-855.

[36] J. C. Martin, W. J. Moyce, An experimental study of the collapse of liquid columns on a rigid horizontal plane, Philosophical Transactions of the Royal Society of London. Series A, Mathematical and Physical Sciences 244 (1) (1952) 312-324. 